\title{
Coating thickness and coverage effects on the forces between silica nanoparticles in water
}

\author{
K. Michael Salerno, ${ }^{1}$ Ahmed E. Ismail, ${ }^{2}$ J. Matthew D. Lane, ${ }^{1}$ and Gary S. Grest ${ }^{1}$ \\ 1) Sandia National Laboratories, Albuquerque, NM 87185, \\ United States \\ 2) Aachener Verfahrenstechnik: Molecular Simulations and Transformations, \\ Faculty of Mechanical Engineering, RWTH Aachen University, D-52056 Aachen, \\ Germany
}

(Dated: 9 October 2018)

The structure and interactions of coated silica nanoparticles have been studied in water using molecular dynamics simulations. For $5 \mathrm{~nm}$ diameter amorphous silica nanoparticles we studied the effects of varying the chain length and grafting density of polyethylene oxide (PEO) on the nanoparticle coating's shape and on nanoparticlenanoparticle effective forces. For short ligands of length $n=6$ and $n=20$ repeat units, the coatings are radially symmetric while for longer chains $(n=100)$ the coatings are highly anisotropic. This anisotropy appears to be governed primarily by chain length, with coverage playing a secondary role. For the largest chain lengths considered, the strongly anisotropic shape makes fitting to a simple radial force model impossible. For shorter ligands, where the coatings are isotropic, we found that the force between pairs of nanoparticles is purely repulsive and can be fit to the form $\left(R / 2 r_{\text {core }}-1\right)^{-b}$ where $R$ is the separation between the center of the nanoparticles, $r_{\text {core }}$ is the radius of the silica core, and $b$ is measured to be between 2.3 and 4.1 . 


\section{INTRODUCTION}

Coated nanoparticles have attracted significant industrial and scientific interest because of their wide range of potential uses, including biomedical applications such as drug delivery ${ }^{1}$ and toxin detection, ${ }^{2}$ as well as more traditional commercial uses such as fillers, dispersants, and surfactants in solution. ${ }^{3}$ Although the use of nanoparticles continues to increase, our understanding of the molecular mechanisms through which nanoparticles interact with one another and with their environments has not kept pace. It is understood that nanoparticle coatings can be used to control aggregation and promote solvation in a processing medium. Only recently, however, have the effects of variables such as the length, grafting density, and structure of the polymer chain coating been directly linked to the dynamics of and interactions between nanoparticles. ${ }^{4-6}$

Experimental study of the stability of nanoparticles in dilute and concentrated polymer solutions have shown that the length of ligands grafted onto nanoparticles, compared to the size of the solvent, has a major effect on stability. ${ }^{7}$ The structure of brushes, including block copolymers, have been explored with particles produced by techniques such as reversible addition-fragmentation chain transfer polymerization. ${ }^{8}$ Rheometry and dynamic light scattering measurements have been used to study viscosity and optical effects on aqueous dispersions of polyethylene oxide (PEO) coated silica. ${ }^{9}$ Recent work in this area includes the use of plasmon rulers to measure interfacial interactions in nanoparticles, ${ }^{10}$ as well as low-energy ion scattering ${ }^{11}$ and infrared spectroscopy ${ }^{12}$ to explore the structure of ionic liquids containing nanoparticles.

The forces and potentials describing the interactions of nanoparticles in solution have also been studied using computational approaches. ${ }^{13}$ For instance, bare colloidal nanoparticles have been explored in Lennard-Jones ${ }^{14-16}$ and $n$-decane ${ }^{17}$ solvents, as well as in electrolyte solutions. ${ }^{18,19}$ Forces between coated nanoparticles have been studied in coarse-grained models using Monte-Carlo methods ${ }^{20}$ as well as in fully atomistic molecular dynamics (MD) simulations of PbSe nanoparticles capped with aliphatic chains ${ }^{21}$ and silica nanoparticles with PEO ligands. ${ }^{22}$ MD simulations have also been used to study nanoparticle monolayers at the surface of monomeric and polymeric Lennard-Jones liquids, ${ }^{23}$ and their mechanical properties in a self-assembled monolayer. ${ }^{24}$ Larger assemblies of particles have also been considered. For instance, Lin et al. ${ }^{25}$ have considered aggregation in coarse-grained models of 
functionalized gold nanoparticles. Other groups have examined the roles of functionalization in silica-polystyrene systems using both coarse-grained ${ }^{26}$ and atomistic ${ }^{27,28}$ models.

There have also been recent theoretical developments in the treatment of certain nanoparticle systems. Lin and co-workers have developed a model for the force required to remove a nanoparticle from a substrate. ${ }^{29}$ Similarly, mean-field theory has been used to measure how confinement effects the interactions between assemblies of nanoparticles. ${ }^{30}$ Other work in this area has focused on the dynamics of large groups of nanoparticles, treating them as coarse-grained particles using both Brownian dynamics ${ }^{31}$ and molecular dynamics. ${ }^{32,33}$ Lalatonne et al. ${ }^{34}$ have explored the role of dispersion versus dipolar forces in the organization of

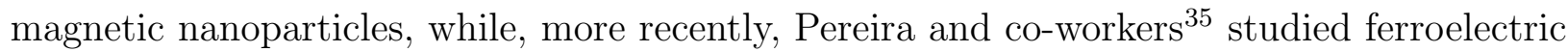
nanoparticles embedded in a nematic liquid crystal.

In our previous work on coated silica nanoparticles ${ }^{22}$ we examined the behavior of functionalized silica nanoparticles approaching either a solid wall or another nanoparticle. For the approach of a single particle toward a wall, the results were shown to be in agreement with the theoretical solution of Brenner. ${ }^{36}$ For the approach of two particles, the results showed that the forces between particles decreases monotonically with increasing distance. This is in notable contrast to results for bare particles, which show strong oscillations in the sign and magnitude of the forces between particles for small separations. ${ }^{15,16}$

Our previous work considered only a single grafting density and chain length in both equilibrium and non-equilibrium conditions. ${ }^{22}$ In this paper, we focus solely on equilibrium conditions but extend our analysis to include the effects of varying the grafting density of ligands, as well as the length of ligands attached to the surface. We show that both of these factors affect the spatial extent of the force interactions between nanoparticles, and combine to determine the effective radius of the nanoparticle. In addition, we address the issue of anisotropy in the structure of coated nanoparticles, showing that ligand length plays the central role in controlling the level of anisotropy in the ligand coating.

Most coarse-grained potential models generally assume that coated spherical cores will themselves be spherical. However, for ligand lengths comparable to the core radius, Lane and Grest have shown previously that asymmetry forms spontaneously ${ }^{37}$ and strongly influences the nanoparticle-nanoparticle interactions and assembly. ${ }^{38}$ Previous experimental and simulation results have shown that for low-density or inhomogeneous graftings anistropic structures can self-assemble. ${ }^{4,39}$ Our simulations do not probe this regime, but instead fo- 
cus on nanoparticle anisotropy in the dense grafting regime. We show, in this work, cases where nanoparticle asymmetry produces interactions which cannot be described via simple radially-dependent potential functions. We limit our attention in the present work to water, which acts as a good solvent for PEO. We have previously reported on the significant effect solvents can have on the structure of polymer coatings. ${ }^{37,40}$

We discuss the basic system studied as well as simulation methodology in Section II. We present results, including measures of effective particle radius and particle anisotropy in Section III, and then discuss how these measurements relate to and inform our measurements of interparticle forces. A summary of findings and some conclusions are presented in Section IV.

\section{METHODOLOGY}

We modeled $5 \mathrm{~nm}$ diameter amorphous silica nanoparticles which had been cut from a sample of bulk amorphous silica and annealed to produce a surface hydroxyl concentration of 4.20 sites per $\mathrm{nm}^{2}$, consistent with experimental values at $300 \mathrm{~K} .^{41}$ The bulk silica was generated from a melt-quench process similar to the method of Lorenz et al. ${ }^{42}$ A passivating coating of methyl-terminated PEO ligands $\left(\mathrm{Si}(\mathrm{OH})_{3} \mathrm{CH}_{2}\left(\mathrm{CH}_{2} \mathrm{CH}_{2} \mathrm{O}\right)_{n}\left(\mathrm{CH}_{3}\right)\right)$ was then attached via chemisorption using trisilanol functional groups in place of selected hydroxyl groups found at the nanoparticle surface. The chains were added one after another, each oriented to point radially away from the surface. If necessary, the chains were rotated about their long axis until the bulky trisilanol head group did not overlap the silica core atoms. Chemisorption sites were selected to be maximally spaced on the sphere from previously placed chains.

The lengths of the ligands studied were $n=6$, 20, and 100 PEO repeat units. For $n=6$, we studied grafting densities $\sigma=1,2$, and 3 chain $/ \mathrm{nm}^{2}$ or 79,157 , and 234 chains, respectively, per 5 nm-diameter nanoparticle. For $n=20$, grafting densities $\sigma=1$ and 2 chain $/ \mathrm{nm}^{2}$ were studied, while for the longest ligands $(n=100), \sigma=0.2$ and $0.5 \mathrm{chain} / \mathrm{nm}^{2}$ were considered, corresponding to 16 or 39 ligands per nanoparticle. These densities are

consistent with previous experimental measurements. ${ }^{43}$ Examples of silica nanoparticles with length $n=6$ and $n=20$ ligands and grafting density 2 chain $/ \mathrm{nm}^{2}$ are shown in Fig. 1 .

Atom interactions were modeled using all-atom force fields developed by Smith et al. for 

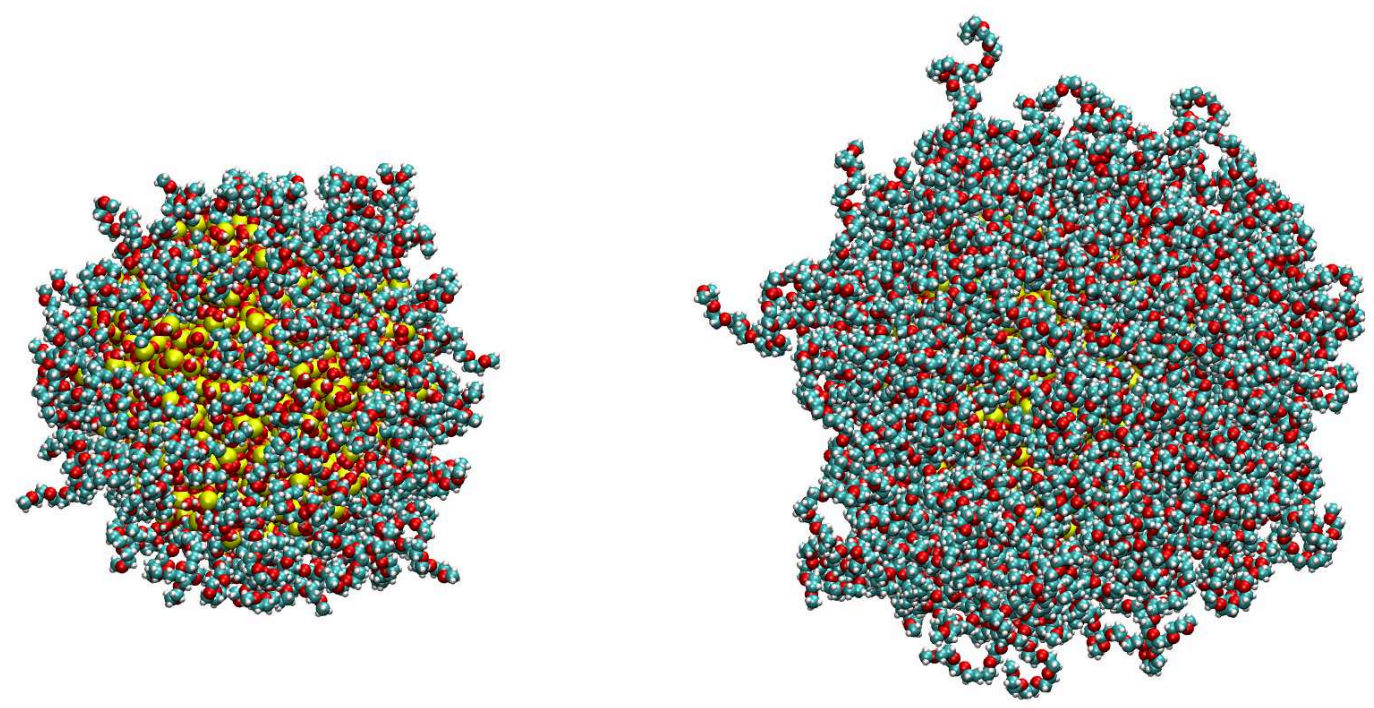

FIG. 1. Representative view of $5 \mathrm{~nm}$ diameter silica nanoparticles functionalized by $n=6$ (left) and $n=20$ (right) PEO ligands with a grafting density of 2 chain $/ \mathrm{nm}^{2}$. Water molecules are excluded for clarity.

both the $\mathrm{PEO}^{44}$ and the silica interactions, ${ }^{45}$ and the TIP4P $/ 2005$ model for water. ${ }^{46,47}$ While Smith et al. provide explicit parameters for the PEO-water interactions, ${ }^{44}$ the parameters for the attractive portion of the Buckingham potentials for the interactions between silica and water were fitted to a Lennard-Jones potential and then combined with the TIP4P potential using Lorentz-Berthelot mixing rules, following the methodology of Ref. 48.

All simulations were carried out using the LAMMPS molecular dynamics code. ${ }^{49}$ The numerical integration was performed using the velocity-Verlet algorithm with a time step of $\delta t=1 \mathrm{fs}$. Lennard-Jones interactions are truncated at $1.2 \mathrm{~nm}$. Long-range Coulomb 
interactions were calculated using the PPPM method with precision $10^{-4} .50$ The nanoparticle core, consisting of the silica molecules plus the termination of each PEO ligand bonded to the silica, was treated as a rigid body, while the bond lengths and bond angles of the water were constrained using the SHAKE algorithm. ${ }^{51}$

To build the nanoparticle-water composite systems, we first equilibrated a rectangular cell of water at $300 \mathrm{~K}$ and $1 \mathrm{~atm}$ for $1 \mathrm{~ns}$. For the $n=6$ and $n=20$ nanoparticles, the initial unit cell size was approximately $13.0 \mathrm{~nm} \times 13.0 \mathrm{~nm} \times 11.5 \mathrm{~nm}$; for the $n=100$ nanoparticles, the unit cell size was $26.0 \mathrm{~nm} \times 26.0 \mathrm{~nm} \times 23.0 \mathrm{~nm}$. The composite system was created by inserting the nanoparticle into a spherical hole cut in the periodic bulk solvent. The resulting system was then equilibrated in the NPT ensemble by applying a Nosé-Hoover barostat ${ }^{52}$ at $300 \mathrm{~K}$ and $1 \mathrm{~atm}$ pressure for at least $0.5 \mathrm{~ns}$ with a thermostat time constant of $0.1 \mathrm{ps}$ and a barostat time constant of $1 \mathrm{ps}$.

Coating structure and anisotropy calculations were made from longer single-particle simulations in the NVT ensemble using a Nosé-Hoover thermostat with time constant 0.1 ps. The center of mass of the rigid core was held fixed during these simulations, though rotations about the center of mass were permitted. Atoms in the PEO coating and water molecules were allowed to move freely. The configuration of the particle ligands and the surrounding water was stored every 5 ps during a 4 ns sampling period.

Nanoparticle-nanoparticle force calculations were made from two-particle systems created by replicating the simulation. After an additional $1 \mathrm{~ns}$ equilibration, the particles were brought together by displacing the center-of-mass of each core at a rate of $25 \mathrm{~nm} / \mathrm{ns}$ toward the center of the box. Positions of all atoms were stored every 10 ps, corresponding to a total net displacement of $0.5 \mathrm{~nm}$. The approach calculation proceeded until the particle cores were nearly in contact.

The configurations obtained from this "approach" simulation were not directly used to measure the forces. Instead, we followed the approach used to produce the static configurations in Ref. 22. Each of the configurations produced from the above run was separately simulated using an $N V T$ ensemble with the center-of-mass of each nanoparticle core frozen. The system was then equilibrated for $1 \mathrm{~ns}$, followed by a sampling run of between $3 \mathrm{~ns}$ and $6 \mathrm{~ns}$ per system. The force between particles was determined by summing all forces on atoms in each core whether exerted by the other particle, the surrounding water, or by the PEO ligands. Forces were calculated at each time step and averaged over $50 \mathrm{fs}$ throughout the 
sampling period.

\section{RESULTS AND DISCUSSION}

\section{A. Nanoparticle Structure}

Examining first the PEO ligand structure, we construct a radial mass density profile $\rho(r)$ for the PEO ligands as a function of distance from the center of mass of the silica particle. The maximum density, $\rho_{\max }$ is used to normalize the density profile. Figure 2 (a) shows the resulting normalized density profiles $\rho^{*}(r)=\rho(r) / \rho_{\max }$ for different brush lengths and grafting densities. From the data in this figure we estimate the half-maximum radius $r_{h}$ as the largest distance at which the normalized density $\rho^{*}\left(r_{h}\right)=0.5$. As in previous studies of nanoparticle ligands in good solvents, ${ }^{40}$ we find that the ligand density is a good measure of the ligand extension into the solvent. There is a notable shoulder in all curves except for the $n=6$ chains at low coverage $\sigma=1-2$ chain $/ \mathrm{nm}^{2}$. This shoulder corresponds to the first carbon - oxygen sequence along the PEO backbone, with its increased mass density. The absence of the feature from the short, low-density configurations indicates that in these systems the PEO chains are able to orient more freely, even very near to the surface.

Examining the half-maximum radii shown in Table I we see that $r_{h}$ increases monotonically with increasing coverage density for a given ligand length. This can be explained by the radial orientation of the chains caused by packing effects. Comparison of values between different ligand lengths, however, suggests that it is the total number of repeat units in the coating that determines the half-maximum radius: note that the half-maximum radius remains unchanged when changing between configurations with approximately the same total number of repeat units, such as $n=20, \sigma=1$ chain $/ \mathrm{nm}^{2}$ and $n=100, \sigma=0.2 \mathrm{chain} / \mathrm{nm}^{2}$ or $n=20, \sigma=2$ chain $/ \mathrm{nm}^{2}$ and $n=100, \sigma=0.5$ chain $/ \mathrm{nm}^{2}$.

Another measure of ligand conformation is the radius of maximum extent $r_{\max }$, which measures the distance the ligands extend beyond the nanoparticle surface. Values of $r_{\max }$ are reported in Table I. For the $n=6$ nanoparticles, the maximum extent of the ligands is between $1.8 \mathrm{~nm}$ and $2.2 \mathrm{~nm}$. Given that the backbone end-to-end length is approximately $2.2 \mathrm{~nm}$, this implies that the ligands are largely uncoiled, with a greater fraction uncoiled at higher grafting densities. For the $n=20$ system the ligands extend between 2.3 and 2.9 

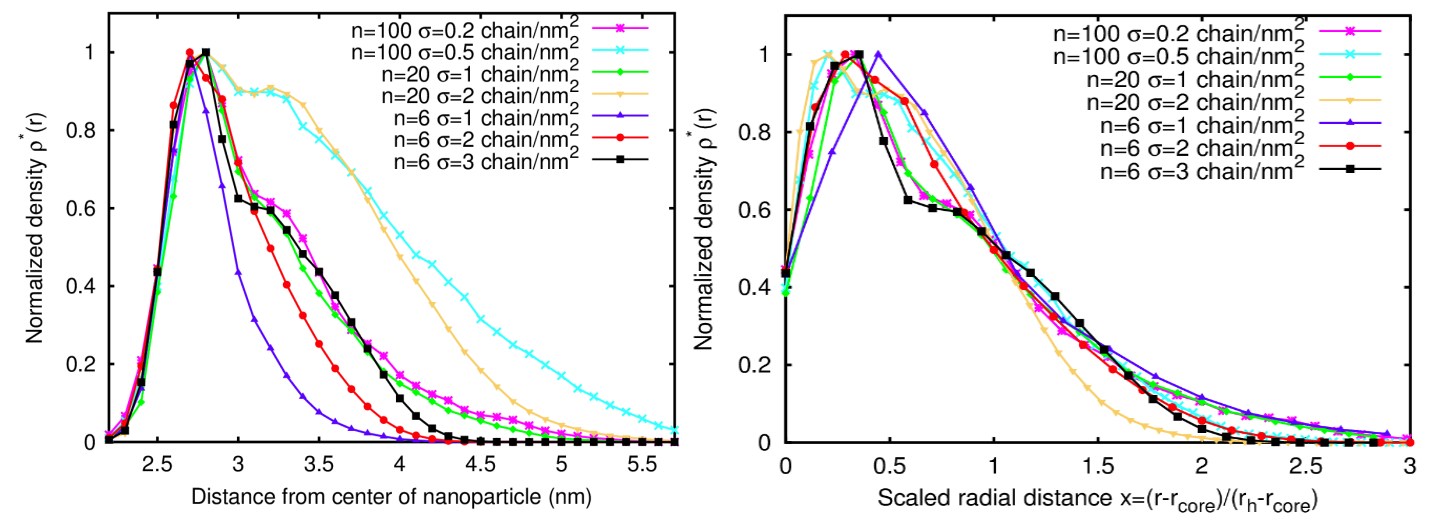

FIG. 2. (a) Normalized brush density $\rho^{*}(r)=\rho(r) / \rho_{\max }$ as a function of distance from the particle center (in $\mathrm{nm}$ ) for ligand length $n=6$ systems with grafting densities $\sigma=3$ (black $\mathbf{\square}), 2$ (red $\bullet$ ), and 1 chain $/ \mathrm{nm}^{2}$ (blue $\boldsymbol{\Delta}$ ); for $n=20$ systems with densities $\sigma=2$ (yellow $\mathbf{\nabla}$ ) and 1 chain $/ \mathrm{nm}^{2}$ (green $\checkmark$ ); and for $n=100$ systems for $0.5\left(\right.$ cyan $\times$ ) and 0.2 chain $/ \mathrm{nm}^{2}$ (magenta $*$ ). (b) The same normalized brush density plotted as a function of scaled distance $x=\left(r-r_{\text {core }}\right) /\left(r_{h}-r_{\text {core }}\right)$.

nm. This is somewhat larger than the expected end-to-end distance of a random coil, but much shorter than the distance along the uncoiled backbone. This is consistent with the ligands near the particle core being partially ordered, with a transition to a random coil-like orientation further from the core, closer to the coating surface. Finally, for the $n=100$ ligands, the maximum extent is comparable in magnitude to the end-to-end distance in solution. ${ }^{53}$ This suggests that for longer ligands with low grafting densities the ligands may be able to attain a coil-like orientation in space. At higher densities, however, there may be interactions between the chains, leading to slightly more ordered chains and thus a larger maximum extent.

From the half-maximum radius we may define a dimensionless radial coordinate

$$
x=\frac{\left(r-r_{\text {core }}\right)}{\left(r_{h}-r_{\text {core }}\right)},
$$

where $r_{\text {core }}=2.50 \mathrm{~nm}$ is the radius of the silica core. This coordinate can be used to compare the PEO density profiles across different ligand length and coverage configurations. Figure 2 (b) shows the normalized PEO density $\rho^{*}(r)$ as a function of $x$. The coordinate $x$ captures much of the variation in radial ligand density for different chain configurations, although there is some variation in the scaled curves. The location of the density maximum moves 
TABLE I. Characteristic radii $r_{h}$ and $r_{\max }$, relative standard deviations of the grafting, chain, and methyl densities as a function of ligand length and grafting density, and ratio of largest to smallest moment of inertia eigenvalues

\begin{tabular}{cccccccc}
\hline \hline Ligand length & $\sigma$ & $r_{h}$ & $r_{\max }$ & \multicolumn{2}{c}{ Relative standard deviation (\%) } & \multicolumn{2}{c}{ Eigenvalue Ratio } \\
\cline { 5 - 6 }$n$ & $\left(\right.$ chain $\left./ \mathrm{nm}^{2}\right)$ & $(\mathrm{nm})$ & $(\mathrm{nm})$ & Grafting density Coating density Methyl density & $\lambda_{\max } / \lambda_{\min }$ \\
\hline 6 & 1 & 2.95 & 4.35 & 10.5 & 10.3 & 17.0 & 1.15 \\
6 & 2 & 3.20 & 4.35 & 10.2 & 8.6 & 10.1 & 1.12 \\
6 & 3 & 3.35 & 4.65 & 8.6 & 6.3 & 8.1 & 1.10 \\
20 & 1 & 3.35 & 5.75 & 10.4 & 13.4 & 18.5 & 1.21 \\
20 & 2 & 3.95 & 5.95 & 10.3 & 8.7 & 15.1 & 1.19 \\
100 & 0.2 & 3.35 & 5.30 & 30.7 & 28.3 & 59.8 & 1.70 \\
100 & 0.5 & 3.95 & 6.50 & 15.4 & 25.5 & 30.4 & 1.75 \\
\hline \hline
\end{tabular}

closer to the particle surface as $r_{h}$ increases. This is expected from the nearly uniform density maximum in Fig. 2 (a) and results in part from the choice of $r_{\text {core }}$. For $x>2$ the tail of the density profile is systematically depressed with increasing grafting density. This variation highlights the limitations of describing the complex interplay of the competing grafting density and chain length effects with the simple $r_{h}$ length scale.

We also examine the density of water as a function of distance from the nanoparticle center. In our previous simulations of PEO and water on a flat substrate, ${ }^{54}$ we have shown that water penetrates readily even into relatively dense polymer brushes. Other simulations of gold nanoclusters with PEO groups along the ligands found that water readily penetrates into such ligands. ${ }^{55}$ Thus, a comparison of the water density as a function of radius versus the bulk density can provide information regarding the structure of the PEO coating around the silica core. Figure 3 shows the density of water molecules as a function of distance to the nanoparticle center of mass.

From Fig. 3 we see that, as for the half-maximum radius, the total number of repeat units grafted onto the particle appears to be the most important factor in determining the water density profile: for instance, there is relatively little difference between the systems with $n=6$ at $3 \mathrm{chain} / \mathrm{nm}^{2}, n=20$ at $1 \mathrm{chain} / \mathrm{nm}^{2}$, and $n=100$ at $0.2 \mathrm{chain} / \mathrm{nm}^{2}$, containing approximately the same total number of repeat units. The primary difference between the 


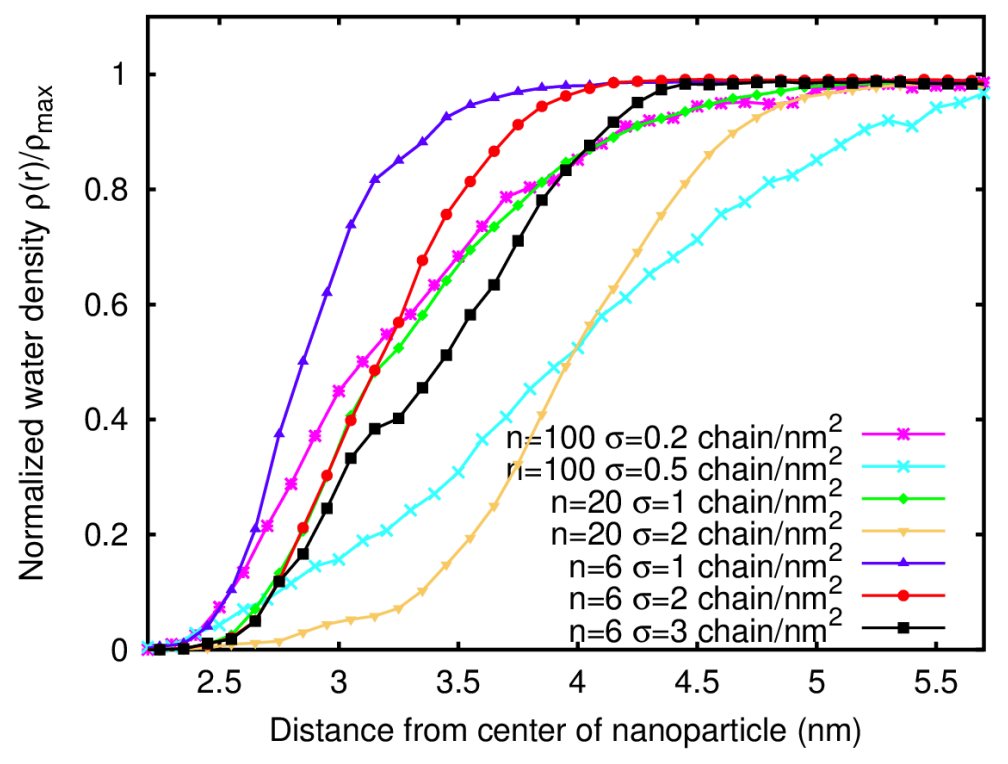

FIG. 3. Normalized water density as a function of distance from the particle center (in nm) for chain length $n=6$ systems for grafting densities of 3 (black $\boldsymbol{\square}$ ), 2 (red •), and 1 chain/nm² (blue $\boldsymbol{\Delta}$ ); for $n=20$ systems for densities of 2 (yellow $\boldsymbol{\nabla}$ ) and 1 (green $\bullet$ ) chain $/ \mathrm{nm}^{2}$; and for $n=100$ systems for $0.5($ cyan $\times)$ and $0.2($ magenta $*)$ chain $/ \mathrm{nm}^{2}$.

curves is that the radius beyond which the density of water equals its bulk density increases with $n$. 


\section{B. Particle anisotropy}

In some cases, a nanoparticle's coating ligands are observed to orient and close pack together. This ordering of the chains can be observed in spatial variations of the local coating densities. We refer to these spatial variations and the oriented ligands that cause them as characteristics of anisotropic coatings. We describe, here, a method for quantifying the degree of anisotropy in coatings and show later that, in its extreme, this asymmetry can destroy the radial symmetry of the interaction forces.

We characterize the surface inhomogeneity of each particle's coating by the standard deviation in the local chain density over the surface. The local density of PEO chain atoms was calculated at 2000 overlapping patches equally spaced over the sphere's surface. ${ }^{56}$ An atom was determined to be in an angular patch about a point if it was inside a cone with vertex at the nanoparticle center and axis passing through said point on the surface. Cones with $90^{\circ}$ opening angles were used, giving each patch an area of $11.5 \mathrm{~nm}^{2}$ at the surface, or approximately $15 \%$ of the total surface area of the nanoparticle. This patch size was selected because it is a reasonable contact patch for two approaching particles. From the collection of patch densities a mean and standard deviation can be calculated. The mean simply returns the overall density of the coating. The standard deviation is a measure of how this density varies locally on the surface, a measure of asymmetry. In order to compare across particles with different grafting densities, we report the relative standard deviation i.e. the standard deviation as a percentage of the mean. This calculation was performed for the grafting points as well as the total coating and the terminal methyl groups.

The relative standard deviation (RSD) of the local coating, grafting point, and methyl group densities are shown in Table I for the different particles studied. Several trends are evident. First, the relative standard deviation in the grafting density decreases slightly with increasing grafting density, as more densely packed coatings are less likely to have gaps in the coatings which increase the RSDs of the patch densities. We see that for short chains the coating asymmetry is somewhat lower than the grafting asymmetry. However, for lower grafting density and longer chains both the coating asymmetry and the methyl asymmetry can be significantly higher than the grafting asymmetry. This indicates that long chains

orient and close pack more strongly than shorter chains and this leads to more anisotropic coatings. This effect is likely to be dramatic for coating ligands in a poor solvent, leading 
to even greater anisotropy.

Particle anisotropy can also be quantified using the eigenvalues of the moment of inertia tensor. The ratio of the largest to smallest eigenvalues compares the smallest and largest axes of the particle, giving a sense asymmetry or anisotropy. The ratio will equal one for spherically symmetric objects, while it will be larger than one for objects which are not spherically symmetric. Eigenvalue ratios for the different particles studied are listed in Table I. The trend of increasing anisotropy with increasing chain length is clearly reflected. Particles with higher grafting density also tend to be slightly more isotropic, as expected, though this trend breaks down for the most anisotropic particles.

An example of a highly anisotropic particle can be seen for the $5 \mathrm{~nm}$ silica nanoparticle coated by $n=100$ chains at grafting density 0.5 chain $/ \mathrm{nm}^{2}$ shown in Fig. 4 . The anisotropy of the grafted ligands for this particle contrasts with the spherically symmetric particles shown in Fig. 1. For asymmetric particles, the function representing the force between particles is no longer a function only of the interparticle distance, since the effective radii of the particles may vary significantly with direction. For this reason, the $n=100$ nanoparticles were excluded from the analysis of forces below. Experimental measurements of PEO grafted onto silica nanoparticles find that for long chains surface densities consistent with our most anisotropic particles are possible i.e. $\sigma=0.2$ chain $/ \mathrm{nm}^{2}$ for $n \approx 100,{ }^{43}$ indicating that such anisotropic coatings may be found in experimentally realizable systems.

\section{Forces between nanoparticles}

Using the two-particle systems described in Section II we measure the forces between particles at a number of radial separations for the $n=6$ and $n=20$ systems. As described above, we limit our analysis to these systems because we assume a spherically symmetric force, which is not a valid assumption for the longer chains. As expected, we find that the interparticle forces decrease as a function of radial separation $R$. At small separations interparticle forces rapidly exceed those found in equilibrium systems or systems under shear. The location of the divergence is set by the nanoparticle radius, while the scale of the interaction depends on both the chain length $n$ and the grafting density $\sigma$. We can test whether different nanoparticle configurations collapse on a single force curve using the scaling based on the half-maximum radius. 


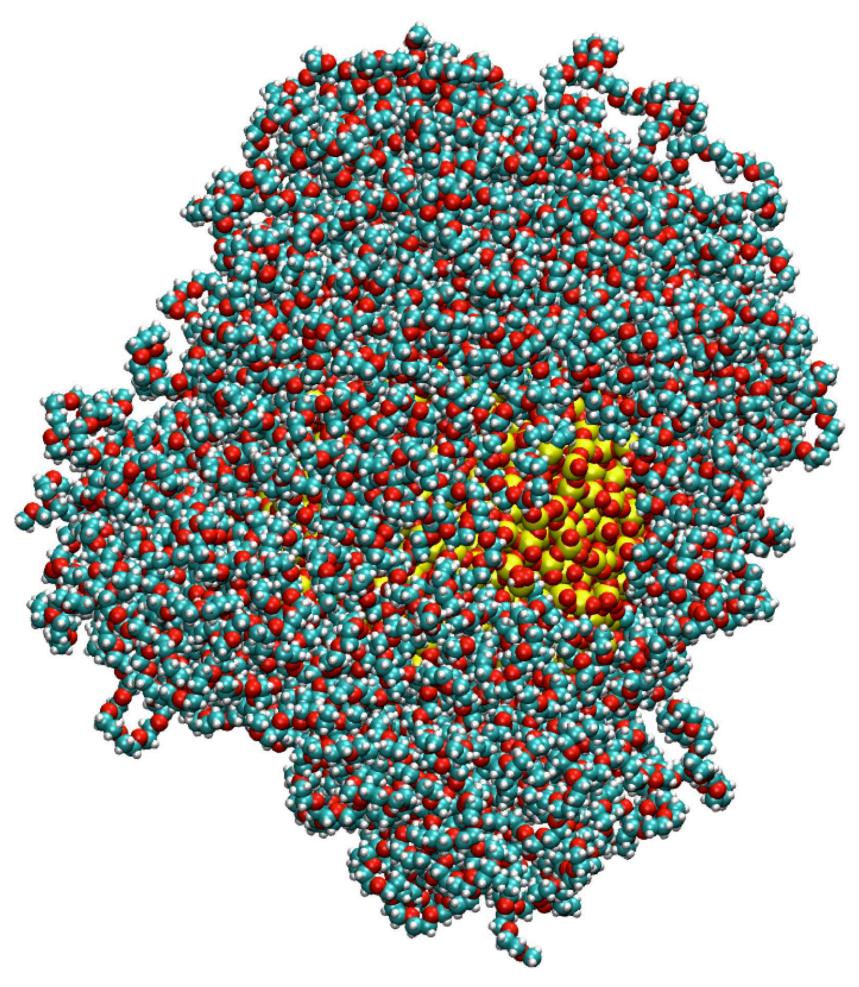

FIG. 4. Sample image of a $5 \mathrm{~nm}$ silica nanoparticle, coated with PEO of 100 repeat units at a grafting density of 0.5 chain $/ \mathrm{nm}^{2}$.

For all chain lengths we find that the forces are purely repulsive and depend strongly upon the grafting density. Similarly, we find that for a fixed grafting density, the interparticle force also depends on chain length. For particles with a low grafting density of short chains $(n=6$, $\sigma=1$ chain $/ \mathrm{nm}^{2}$ ), the forces are below $1 \mathrm{nN}$ even when there is only a $0.5 \mathrm{~nm}$ gap between the silica surfaces of the particles. For chains with $n=20$ at higher grafting density, the interparticle forces can remain non-zero out to particle separations greater than $9 \mathrm{~nm}$. For both short and long chain particles the magnitude of the equilibrium force appears to fall off to zero at a distance between $2 r_{h}$ and $2 r_{\max }$.

Additional insight can be gained from plotting the force curves against

$$
X=\frac{\left(R-2 r_{\text {core }}\right)}{2\left(r_{h}-r_{\text {core }}\right)},
$$




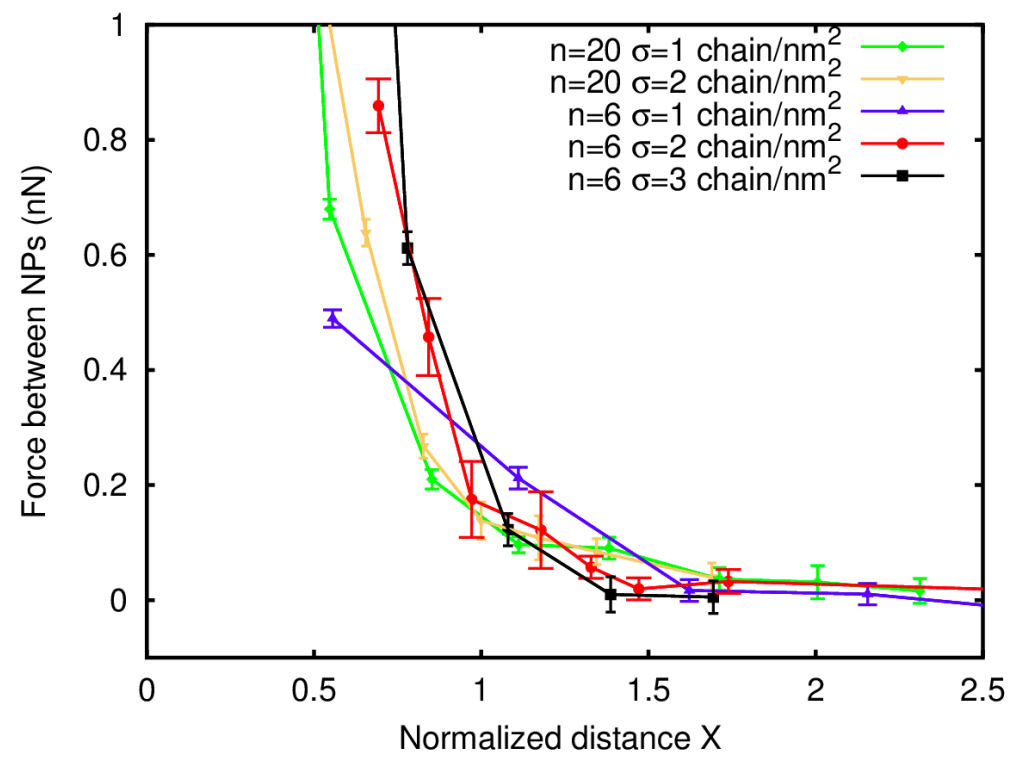

FIG. 5. Equilibrium forces between functionalized nanoparticles as a function of shifted and scaled radial separation $X=\left(R-2 r_{\text {core }}\right) / 2\left(r_{h}-r_{\text {core }}\right)$ for chain length $n=6$ systems for grafting densities of 3 (black $\mathbf{\square}$ ), 2 (red $\bullet$ ), and 1 chain $/ \mathrm{nm}^{2}$ (blue $\boldsymbol{\Delta}$ ); and for $n=20$ systems for densities of 2 (yellow $\mathbf{\nabla}$ ) and 1 (green $\bullet$ ) chain $/ \mathrm{nm}^{2}$.

the distance between the surfaces of the two particles normalized by the width of both brushes, as shown in Fig. 5. When this scaling is taken into account, it appears that all of the systems follow the same trend: large forces for $X<1$, followed by forces approaching zero between $1<X<2$. There is some variation between the scaled curves, indicating that the interparticle force is not entirely governed by half-maximum radius $r_{h}$. In particular, the system with the fewest total repeat units, $n=6, \sigma=1$ chain $/ \mathrm{nm}^{2}$ appears to have a much 


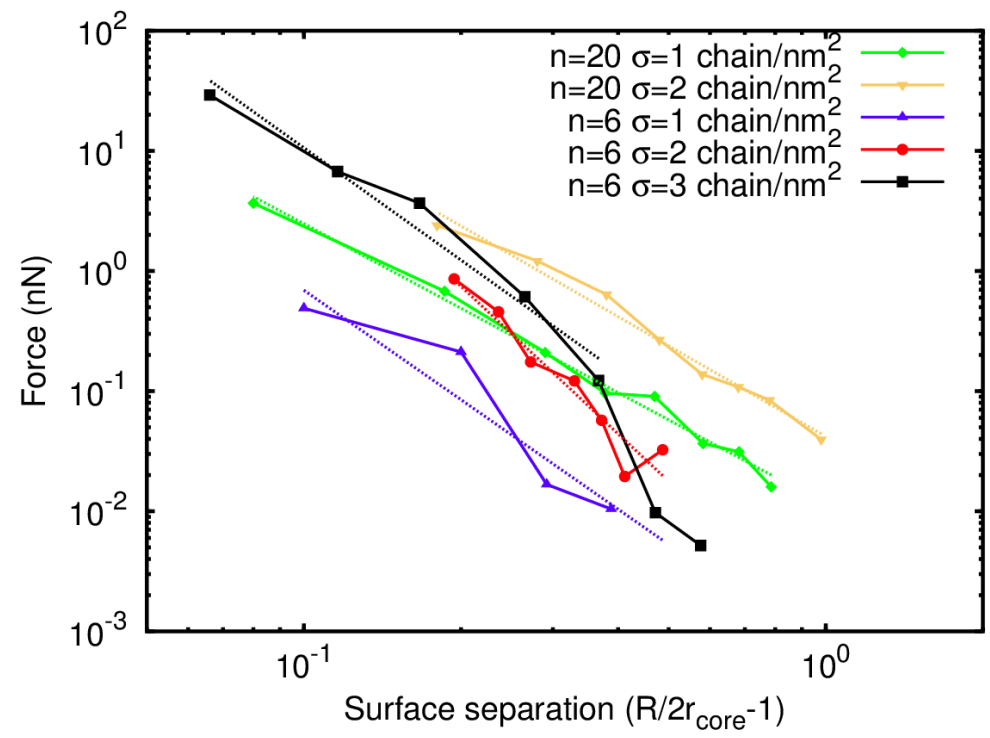

FIG. 6. Force curves as a function of shifted particle separation $\left(R / 2 r_{\text {core }}-1\right)$ for the studied systems plotted on log-log axes. Fits are shown as dashed lines, with parameters listed in Table II.

softer force curve. For the denser $n=6$ and $n=20$ systems the scaled force curves agree and rapidly increase as $X$ is reduced from roughly 1.5 to 0.5 .

Shifted force curves can be plotted on log-log axes in order to compare the steepness of the slope increase. Because a divergence is expected at radial separation $R=5.0 \mathrm{~nm}$, the curves have been shifted by the nanoparticle core diameter. These curves are shown in Fig. 6 and can be fit to the form of a prefactor times a power of the surface separation $F(R)=$ $A\left(R / 2 r_{\text {core }}-1\right)^{-b}$. For the particles studied, we find $6.5 \times 10^{-4} \mathrm{nN}<A<4.3 \times 10^{-2} \mathrm{nN}$ and $2.3<b<4.1$. These fits are shown as dashed lines in Fig 6 , with parameters recorded 
in Table II. Although the range of data is small, the trend indicates that the slope is steeper at smaller $(n=6)$ chain lengths, fitting the intuitive picture that short chains lead to stiffer interactions. The trend is also consistent with the geometric picture of longer chains containing both coiled and uncoiled conformations, leading to a softer interaction.

\section{Second Virial Coefficient}

The second virial coefficient is related to the osmotic pressure of a collection of macromolecules in solution, and is directly accessible via multiple experimental measurements. The numerical value of the coefficient can also be calculated from the force or potential between the nanoparticles measured during simulation. ${ }^{13}$ From this calculation we give an estimate of the value of the second virial coefficient for our nanoparticles and compare these estimates with experimental values.

The standard calculation of the osmotic second virial coefficient $A_{2}$ is based on the definition

$$
A_{2}=\frac{2 \pi N_{A}}{M^{2}} \int_{0}^{\infty}\left(1-e^{-U(R) / k T}\right) R^{2} d R
$$

where $U(R)$ is the potential between two interacting particles, $M$ is the molar mass of a nanoparticle, and $N_{A}$ is Avogadro's number. The definition above depends only on the radial distance $R$. As before, this approximation is valid only for the $n=6$ and $n=20$ isotropic particle cases. We calculate the second virial coefficient based on the forces measured in Section IIIC, and determine the interparticle potential as the integral of the force with respect to radial distance $R$. The force can be integrated analytically to produce a potential $U(R)=C_{1} /\left(R / 2 r_{\text {core }}-1\right)^{b-1}+C_{0}$, where $C_{0}$ is chosen so that the potential goes continuously to zero beyond an upper radial cutoff. The potential is considered to be infinite for $R<2 r_{\text {core }}$ and zero for $R$ larger than the upper cutoff.

For the nanoparticle systems studied we need to estimate an upper radial cutoff, beyond which both the force and potential are zero. One estimate $R_{0}$ for the radial cutoff is the point at which the magnitude of the fluctuations in the force are larger than the magnitude of the force itself, so that the force is effectively zero. This occurs for forces less than about $0.05 \mathrm{nN}$ and the large fluctuations below this level can be seen on both the linear and log scales in Figs. 5 and 6 . Another, more conservative estimate of the radial cutoff is $2 r_{\max }$. Because the interparticle forces are caused by steric interactions, the largest particle separation that can 
TABLE II. Fit parameters and values of the osmotic second virial coefficient for different nanoparticle-ligand configurations.

\begin{tabular}{|c|c|c|c|c|c|}
\hline \multicolumn{4}{|c|}{$\left(\right.$ chain $\left./ \mathrm{nm}^{2}\right)\left(\times 10^{-4} \mathrm{nN}\right)$} & $\left(10^{-5} \mathrm{~cm}^{3} \mathrm{~mol} / \mathrm{g}^{2}\right)\left(10^{-5} \mathrm{~cm}^{3} \mathrm{~mol} / \mathrm{g}^{2}\right)$ & $\begin{array}{c}A_{2} \text { from } 2 r_{\max } \\
\left(10^{-5} \mathrm{~cm}^{3} \mathrm{~mol} / \mathrm{g}^{2}\right)\end{array}$ \\
\hline 6 & 1 & 6.5 & 3.0 & 2.1 & 3.8 \\
\hline 6 & 2 & 11 & 4.1 & 2.0 & 3.2 \\
\hline 6 & 3 & 85 & 3.1 & 2.0 & 3.3 \\
\hline 20 & 1 & 110 & 2.3 & 2.0 & 5.6 \\
\hline 20 & 2 & 430 & 2.5 & 2.0 & 4.4 \\
\hline
\end{tabular}

contribute to the integral is $R=2 r_{\max }$. Based on these cutoff radii and the potential $U(R)$, numerical values of the integral in Eq. 3 can be calculated. Both fit values and estimates of the second virial coefficient are recorded in Table II. We note that using $2 r_{\max }$ as an upper cutoff assumes that the functional form of the force and potential do not change beyond where they can be reliably measured with molecular dynamics. Checking the validity of this assumption is a very costly computational task, but the variation of the exact cutoff value does not appear to dramatically change the calculated value of the second virial coefficient.

The osmotic second virial coefficient has been experimentally measured in a number of sterically stabilized nanoparticle systems. Jansen et al. studied silica particles of radius $31 \mathrm{~nm}$ with linear carbon chain ligands in toluene, finding second virial coefficients at room temperature similar in magnitude to those reported here. ${ }^{57}$ More recently, the second virial coefficient of CdSe nanocrystals with grafted trioctylphosphine oxide was measured using membrane osmometry. ${ }^{58}$ For comparable particle sizes $(4.6 \mathrm{~nm}$ diameter) measurements of the virial coefficient varied between $1.5 \times 10^{-5}$ and $7 \times 10^{-5} \mathrm{~cm}^{3} \mathrm{~mol} / \mathrm{g}^{2}$, in line with our measurements. Measurements on slightly different nanoparticle solvent systems also give measurements of similar magnitude, about $2 \times 10^{-5} \mathrm{~cm}^{3} \mathrm{~mol} / \mathrm{g}^{2} .{ }^{59}$

\section{CONCLUSIONS}

We have explored the structure of PEO-coated silica nanoparticles as a function of chain length and grafting density, as well as the interactions between pairs of particles. The total 
mass grafted onto the surface appears to be the most important factor in determining the structure of the particle, as both the density profile and half-maximum radius appear to vary directly with the total number of repeat units. The maximum extent of the chains from the particle surface also increases weakly with grafting density. This suggests that long chains undergo a transition from a primarily linear structure near the grafting point to a more coil-like structure far away from the grafting point.

The force between two nanoparticles was determined as a function of separation for all nanoparticle configurations. As chain size increases, the tendency of the chains to achieve a coil-like conformation increases, influencing the extent of the chains and the half-maximum radius $r_{h}$. As chain length increases the anisotropy of the particle also increases to such an extent that the assumption of a radially symmetric particle breaks down, and the relative orientation of the nanoparticles needs to be considered in describing the interactions between them. When particles are separated by less than twice the half-maximum radius $r_{h}$ of a particle, the forces rapidly increase; for separations greater than about $2.5 r_{h}$, it is difficult to distinguish interactions beyond statistical uncertainty.

The second virial coefficient was calculated from analytical fits of the measured interparticle forces and assumption of a radial cutoff. Coefficient values fell in the range of 1-10 $\times 10^{-5} \mathrm{~cm}^{3} \mathrm{~mol} / \mathrm{g}^{2}$ and are comparable to experimental measurements. For the purely repulsive forces found here, this coefficient represents and effective nanoparticle volume due to the steric interactions of the PEO chains.

\section{ACKNOWLEDGMENTS}

AEI was supported by the Cluster of Excellence "Tailor-Made Fuels from Biomass," which is funded by the Excellence Initiative by the German federal and state governments to promote science and research at German universities. This work was performed, in part, at the Center for Integrated Nanotechnology, a U.S. Department of Energy and Office of Basic Energy Sciences user facility. Sandia National Laboratories is a multi-program laboratory managed and operated by Sandia Corporation, a wholly owned subsidiary of Lockheed

Martin Corporation, for the U.S. Department of Energy's National Nuclear Security Administration under contract DE-AC04-94AL85000. 


\section{REFERENCES}

${ }^{1}$ D. A. Giljohann, D. S. Seferos, W. L. Daniel, M. D. Massich, P. C. Patel, and C. A. Mirkin, Angew. Chim. Int. Ed. 49, 3280 (2010).

${ }^{2}$ H. Uzawa, K. Ohga, Y. Shinozaki, I. Ohsawa, T. Nagatsuka, Y. Seto, and Y. Nishida, Biosens. Bioelectron. 24, 923 (2008).

${ }^{3}$ R. Anselmann, J. Nanoparticle Res. 3, 329 (2001).

${ }^{4}$ P. Akcora, H. Liu, S. K. Kumar, J. Moll, Y. Li, B. C. Benicewicz, L. S. Schadler, D. Acehan, A. Z. Panagiotopoulos, V. Pryamitsyn, V. Ganesan, J. Ilavsky, P. Thiyagarajan, R. H. Colby, and J. F. Douglas, Nature materials 8, 354 (2009).

${ }^{5}$ A. Striolo, Small 3, 628 (2007).

${ }^{6}$ E. Rabani and S. A. Egorov, Nano Letters 2, 69 (2002).

${ }^{7}$ N. Dutta and D. L. Green, Langmuir 24, 5260 (2008).

${ }^{8}$ C. Li and B. C. Benicewicz, Macromolecules 38, 5929 (2005).

${ }^{9}$ Q. Zhang and L. A. Archer, Macromolecules 37, 1928 (2004).

${ }^{10}$ J. H. Yoon and S. Yoon, Langmuir 29, 14772 (2013).

${ }^{11}$ A. Kauling, G. Ebeling, J. Morais, A. Pádua, T. Grehl, H. H. Brongersma, and J. Dupont, Langmuir 29, 14301 (2013).

${ }^{12}$ J.-M. Andanson and A. Baiker, J. Phys. Chem. C 117, 12210 (2013).

${ }^{13}$ C. N. Likos, Physics Reports 348, 267 (2001).

${ }^{14}$ Y. Qin and K. A. Fichthorn, J. Chem. Phys. 119, 9745 (2003).

${ }^{15}$ Y. Qin and K. A. Fichthorn, Phys. Rev. E 73, 020401 (2006).

${ }^{16}$ S. R. Challa and F. van Swol, Phys. Rev. E 73, 016306 (2006).

${ }^{17}$ Y. Qin and K. A. Fichthorn, J. Chem. Phys. 127, 144911 (2007).

${ }^{18}$ S. Jenkins, S. R. Kirk, M. Persson, J. Carlen, and Z. Abbas, J. Chem. Phys. 127, 224711 (2007).

${ }^{19}$ S. Jenkins, S. R. Kirk, M. Persson, J. Carlen, and Z. Abbas, J. Chem. Phys. 128, 164711 (2008).

${ }^{20}$ A. Striolo, Phys. Rev. E 74, 041401 (2006).

${ }^{21}$ A. P. Kaushik and P. Clancy, The Journal of Chemical Physics 136, 114702 (2012).

${ }^{22}$ J. M. D. Lane, A. E. Ismail, M. Chandross, C. D. Lorenz, and G. S. Grest, Phys. Rev. E 79, 050501 (2009). 
${ }^{23}$ S. Cheng and G. S. Grest, J Chem Phys 136, 214702 (2012).

${ }^{24}$ S. S. You, R. Rashkov, P. Kanjanaboos, I. Calderon, M. Meron, H. M. Jaeger, and B. Lin, Langmuir (2013).

${ }^{25}$ J.-Q. Lin, H.-W. Zhang, Z. Chen, Y.-G. Zheng, Z.-Q. Zhang, and H.-F. Ye, The Journal of Physical Chemistry C 115, 18991 (2011).

${ }^{26}$ A. Ghanbari, T. V. M. Ndoro, F. Leroy, M. Rahimi, M. C. Böhm, and F. Müller-Plathe, Macromolecules 45, 572 (2012).

${ }^{27}$ T. V. M. Ndoro, E. Voyiatzis, A. Ghanbari, D. N. Theodorou, M. C. Böhm, and F. MüllerPlathe, Macromolecules 44, 2316 (2011).

${ }^{28}$ T. V. M. Ndoro, M. C. Böhm, and F. Müller-Plathe, Macromolecules 45, 171 (2012).

${ }^{29}$ J. Lin, Y. Lin, and J. Qian, Langmuir (2013).

${ }^{30}$ V. Pryamitsyn and V. Ganesan, J. Chem. Phys. 138, 234905 (2013).

${ }^{31}$ A. S. Almusallam and D. S. Sholl, Nanotechnology 16, S409 (2005).

${ }^{32}$ I. N. Kar'kin, Y. N. Goronstyrev, and L. E. Kar'kina, Phys. Solid State 52, 431 (2010).

${ }^{33}$ R. J. K. Udayana Ranatunga, R. J. B. Kalescky, C. Chiu, and S. O. Nielsen, J. Phys. Chem. C 114, 12151 (2010).

${ }^{34}$ Y. Lalatonne, J. Richardi, and M. P. Pileni, Nature Materials 3, 121 (2004).

${ }^{35}$ M. S. S. Pereira, A. A. Canabarro, I. N. de Olivieira, M. L. Lyra, and L. V. Mirantsev, Eur. Phys. J. E 31, 81 (2010).

${ }^{36}$ H. Brenner, Chem. Eng. Sci. 16, 242 (1961).

${ }^{37}$ J. M. D. Lane and G. S. Grest, Phys Rev Lett 104, 235501 (2010).

${ }^{38}$ J. M. D. Lane and G. S. Grest, Nanoscale (2014).

${ }^{39}$ S. K. Kumar, N. Jouault, B. Benicewicz, and T. Neely, Macromolecules 46, 3199 (2013).

${ }^{40}$ B. L. Peters, J. M. D. Lane, A. E. Ismail, and G. S. Grest, Langmuir 28, 17443 (2012).

${ }^{41}$ L. T. Zhuravlev, Langmuir 3, 316 (1987).

${ }^{42}$ C. D. Lorenz, E. B. Webb III, M. J. Stevens, M. Chandross, and G. S. Grest, Trib. Lett. 19, 93 (2005).

${ }^{43}$ P. Maitra, J. Ding, H. Huang, and S. L. Wunder, Langmuir 19, 8994 (2003).

${ }^{44}$ G. D. Smith, O. Borodin, and D. Bedrov, J. Comp. Chem. 15, 1480 (2002).

${ }^{45}$ J. S. Smith, O. Borodin, G. D. Smith, and E. M. Kober, J. Poly. Sci. 45, 1599 (2007).

${ }^{46}$ W. L. Jorgensen, J. Chandrasekhar, J. D. Madura, R. W. Impey, and M. L. Klein, J. Chem. Phys. 79, 926 (1983). 
${ }^{47}$ J. L. F. Abascal and C. Vega, The Journal of Chemical Physics 123, 234505 (2005).

${ }^{48}$ A. E. Ismail, G. S. Grest, D. R. Heine, M. J. Stevens, and M. Tsige, Macromolecules 42, 3186 (2009).

${ }^{49}$ S. Plimpton, J. Comp. Phys. 117, 1 (1995).

${ }^{50}$ R. W. Hockney and J. W. Eastwood, Computer Simulation Using Particles (Adam HilgerIOP, Bristol, 1988).

${ }^{51}$ J. P. Ryckaert, G. Ciccotti, and H. J. C. Berendsen, J. Comp. Phys. 23, 327 (1977).

${ }^{52}$ D. Frenkel and B. Smit, Understanding Molecular Simulation: From Algorithms to Applications, Computational science series (Elsevier Science, 2001).

${ }^{53}$ G. D. Smith, D. Bedrov, and O. Borodin, Journal of the American Chemical Society 122, $9548(2000)$.

${ }^{54}$ A. E. Ismail, G. S. Grest, and M. J. Stevens, Langmuir 23, 8508 (2007).

${ }^{55}$ A.-C. Yang, C.-I. Weng, and T.-C. Chen, J. Chem. Phys. 135, 034101 (2011).

${ }^{56}$ A. T. Clark and M. Lal, J. Chem. Soc., Faraday Trans. 2 74, 1857 (1978).

${ }^{57}$ J. Jansen, C. de Kruif, and A. Vrij, J. Colloid Interf. Sci. 114, 492 (1986).

${ }^{58}$ A. Striolo, J. Ward, J. M. Prausnitz, W. J. Parak, D. Zanchet, D. Gerion, D. Milliron, and A. P. Alivisatos, J. Phys. Chem. B 106, 5500 (2002).

${ }^{59}$ M. E. Mackay, A. Tuteja, P. M. Duxbury, C. J. Hawker, B. Van Horn, Z. Guan, G. Chen, and R. S. Krishnan, Science 311, 1740 (2006). 